\title{
Longitudinal, Transverse, and Torsional Free Vibrational and Mechanical Behavior of Silicon Nanotubes Using an Atomistic Model
}

\author{
Hassan Shirzadi Jahromi ${ }^{a, b}$ (D), AliReza Setoodeh ${ }^{a^{*}}$ (D) \\ ${ }^{a}$ Shiraz University of Technology, Department of Mechanical and Aerospace Engineering, Shiraz 71555, Iran \\ ${ }^{b}$ Western Michigan University, Department of Mechanical and Aerospace Engineering, Kalamazoo, MI \\ 49008, USA
}

Received: February 21, 2020; Accepted: March 27, 2020

\begin{abstract}
Silicon nanotubes (SiNTs) have been successfully synthesized recently. Despite the wide potential applications of SiNTs, their mechanical properties are rarely reported. In this study, as the first step, the mechanical behavior of clamped-free single-walled silicon nanotubes (SWSiNTs) for both armchair and zigzag structures is investigated by using molecular dynamics (MD) simulations while the inter-atomic forces are described by the Tersoff-Brenner many-body potential energy function. Meanwhile, the results of the total strain energy are used to establish an expression for predicting Young's modulus of the nanotubes. Afterward, the free vibrational analysis including torsional, longitudinal and transverse vibrations behavior of SWSiNTs with different diameters and lengths are investigated to report the corresponding fundamental frequencies as a significant design parameter. In this study, also, an important stress-strain parameter ratio is defined for vibration analyses named dynamic Young's modulus. Thereafter, the natural frequencies that are obtained using the current atomistic model are successfully compared with those evaluated by the continuum mechanics model. It is concluded that the utilized approach can predict the frequencies with reasonable accuracy. Furthermore, the effects of geometry on the natural frequencies for both armchair and zigzag structures are examined. The obtained results provide valuable insights into the vibrational behavior of silicon-based nanotubes.
\end{abstract}

Keywords: Molecular dynamics, Silicon Nanotubes, Vibration analysis, Natural Frequency, Dynamic Young's modulus, Mechanical behavior.

\section{Introduction}

The concept of 'nanotechnology' was proposed by physics Nobel laureate, Richard Feynman in 1959. Since then, nanotechnology has developed quickly and now is a significant technological science. Wide potential applications such as molecular electronics, diagnostic biosensors, and novel scanning microscopy probes ${ }^{1}$, make nanotubes one of the most popular nanofilaments. In 1991, Iijima² introduced carbon nanotubes (CNTs). As CNTs are widely implemented as a structural element, thus study their mechcanical behavior is crusial. The mechanical behavior as one of the most important characteristics of the nanostructures can be theoretically studied using quantum mechanic ${ }^{3-8}$, discrete modeling techniques such as molecular dynamics simulation and continuum mechanics ${ }^{9-15}$. Also, the vibration analysis of single/multi-walled carbon nanotubes in the context of coupled methods using molecular mechanics and finite elements method has been considered ${ }^{16-19}$. Meanwhile, Georgantzinos and Giannopoulos ${ }^{20}$ presented an efficient numerical model for the vibration analysis of single-walled carbon nanotube using finite elements methods. Although, each method has some merits, one of the most efficient approach to simulate the mechanical behavior of nanostructures is molecular dynamics. Studying mechanical behavior of nano materials using molecular dynamics simulation is recognized as one

*e-mail: setoodeh@sutech.ac.ir of the most reliable techniques. Legoas et al..$^{21}$ presented one of the first MD simulation of carbon nanotubes used as gigahertz oscillators, and with the advancement of this method, the researchers employed this method for other nano sructural element with different constituents ${ }^{22-29}$.

Recently, silicon based nanotubes (SiNTs) have been successfully synthesized, and many authors applied molecular dynamics simulations to investigate mechanical properties of SiNTs. In this regard, Bahel and Ramakrishna ${ }^{30}$ calculated the lowest energy structure of silicon nanotubes. Menon and Richter ${ }^{31}$ studied stability of a one dimensional silicon structure. Fagan et al. ${ }^{32}$ presented the stability and thermal behavior of SiNTs. The majority of the computer-aided studies and experimental results discussed in literature ${ }^{33,34}$ concern the basic mechanical properties of Si nanotubes under axial tension. For instance, Jeng et al..$^{35}$ examined the effects of temperature, strain rate, and vacancies on the tensile and fatigue behaviors of silicon-based nanotubes. To the best of authurs' knowledge, a MD-based study with focusing on the vibrational behavior of SiNTs has not been yet presented. On the other hand, due to wide potential application of SiNTs in different industries like medical devices and spesificly as new material for fabricatiing heart valve ${ }^{36}$, it is important to determine the vibrational behavior of these nanotubes. Thus, the main target of this article is to extensively study the vibration characteristics of SiNTs in different directions. 
Consequently, by applying appropriate boundary condition to the region of end atoms, the torsional, longitudinal and transverse vibrations are simulated and the coresponding natural frequencies of the nanotubes as a significat design parameter are predicted. Meanwhile, the variations of natural frequencies with respect to the changes in geometry diameter, length and chirality are exhibited in detail.

\section{MD Modeling}

Molecular dynamics simulation is a powerful technique to explore the microcosmic mechanism of deformation and intrinsic characteristics of a structure at the atomic level. For modelling the mechanical properties of nanostructures and nanoparticles, it is a useful and reliable technique. To model Si nanotube based on MD, one needs to calculate the interatomic forces, the elastic properties, the molecular bond energies, and bond lengths of the Si-Si bond for each time step by solving Newton's Equations. The present study pursues the Tersoff-Brenner many-body potential function ${ }^{37,38}$ to model the Si-Si interactions. Regarding this potential, the electronic structural effects such as $\mathrm{sp}^{3}$ bonding are described via a many-body bond ordering expression. the Tersoff potential parameters for the silicon atoms are extracted from Ref..$^{39}$ to determine the interaction among the atoms. Moreover, the total potential energy for SiNT based on the Tersoff-Brenner potential function is expressed $a^{40}$ :

$$
\sum_{i} E_{i}=\frac{1}{2} \sum_{i \neq j} V_{i j}
$$

With

$$
\begin{gathered}
V_{i j}=f_{c}\left(r_{i j}\right)\left[V_{r e p}\left(r_{i j}\right)+b_{i j} V_{a t t}\left(r_{i j}\right)\right] . \\
b_{i j}=b_{i j}\left(g_{i j}\right) ; g_{i j}=\sum_{k} f_{c}\left(r_{i k}\right) g\left(\theta_{i j k}\right) f\left(r_{i j}-r_{i k}\right) .
\end{gathered}
$$

where $E$ is the total energy of the system, $i, j$ and $k$ denote the atoms, $r_{i j}$ is the distance between pairs adjoining atoms $i$ and $j, V_{i j}$ is the bonding energy, $b_{i j}$ is an empirical bond-order term, $V_{\text {att }}$ and $V_{\text {rep }}$ are the attractive and repulsive parts of the potential, $f_{c}\left(r_{i j}\right)$ denotes the cutoff function, $g\left(\theta_{i j k}\right) f\left(r_{i j}-r_{i k}\right)$ is the number of bound to atom beside the $i j$ bound function, $g_{i j}$ is the coordination, $\theta_{i j k}$ denotes the bond angle between bonds $i j$ and $i k^{41}$. In the present simulations which is performed by LAMMPS largescale atomic/molecular massively parallel simulator) open source package ${ }^{42}$, the $\mathrm{Si}-\mathrm{Si}$ bond is considered equal to $2.245 \dot{A}^{43}$, the model is conducted in canonical ensemble (NVT) at room temperature $(300 K)$ to control the volume and temperature, boundary conditions in $x$ any $y$ directions are considered to be periodic in order to decrease the length effect, the nanotubes are extended axially (i.e. in the $z$ direction) by providing accurate uniaxial stress condition, while the timestep used in this model is kept $1 \mathrm{fs}$ during the simulation. In order to set the relaxation state of the atoms, the conjugate gradient method is employed to reach the equilibrium state. At the beginning of the simulation, the system is relaxed for $50 \mathrm{ps}$, and then the external displacements are applied to elongate the relaxed nanotube. Meanwhile, the velocity Varlet algorithm ${ }^{44}$ is used to solve the equations of motion. This procedure is continued until the rupture happens in the nanotube. The initial configuration strained, and fractured conditions of silicon nanotubes is illustrated in Figure 1.

\section{Stiffness of SiNTs}

The Young's modulus $(Y)$ is calculated using the second derivative of the total energy with respect to strain at the zero strain (i.e. at the equilibrium configuration) according to Equation 4. In this equation, $V_{0}$ denotes the equilibrium volume, $\varepsilon$ is the strain and $E$ stands for the total energy. There is an extreme challenge to determine the value of $V_{0}$. Many researchers define this volume by using a shell-thickness equal to the interlayer spacing in silicene. The value of $V_{0}$ for SiNTs can be also found using LAMMPS molecular dynamics simulator's data.

Figure 2 presents the variation of the strain energy per atom, which is determined by difference between the total energy per atom in strained and unstrained condition. The Young's modulus for each nanotube is determined by fitting a polynomial curve to the corresponding plotted data and using Equation 4.

$$
Y=\frac{1}{V_{0}}\left(\frac{\partial^{2} E}{\partial \varepsilon^{2}}\right)_{\varepsilon=0} .
$$

The Young's modulus of $(10,10)$ SiNT is predicted as $52.56(\mathrm{GPa})$ by the present study which shows a great congruence in compare with the existing data in the literature ${ }^{35}$.

(a)

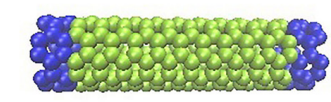

(b)

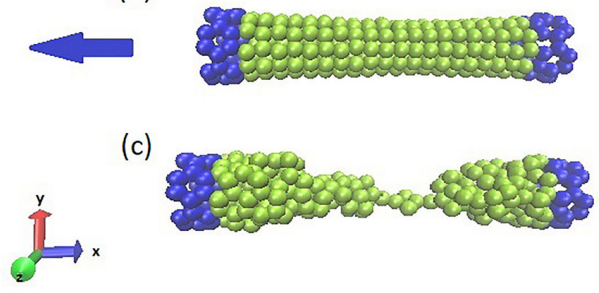

Figure 1. Configuration for tensile process of SiNT. (a) Unstrained, (b) Strained and (c) Fractured conditions.

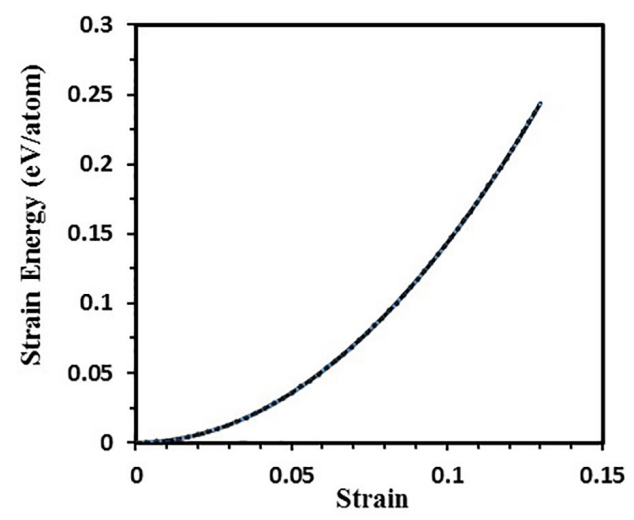

Figure 2. Strain energy values of $(10,10) \mathrm{SiNT}$ as a function of the axial tensile strain from MD simulation. 
After validating the results and verification of the simulated model, Young's modulus of SiNTs with length of $5 \mathrm{~nm}$ and different diameters are determined by the same procedure for both zigzag and armchair structures and illustrated in Table 1. It can be observed that armchair configuration of SiNTs are more stable than zigzag ones due to their higher value of Young's modulus. Moreover, the diameter has a minor effect on the Young's modulus of the nanotubes.

The symmetric per-atom stress tensor of the SiNT is calculated by using "stress/atom" and "compute" commands in Lammps to obtain the desired stress vector. Then, the predicted stress is divided to the volume. Eventually, metric unit conversion is utilized to determine the correct value of the stress. To further study the mechanical behavior of SiNTs, the stress-strain diagrams of armchair and zigzag silicon nanotubes with chiralities of $(5,5),(10,10),(5,0)$, $(10,0)$ are demonstrated respectively in Figures 3 and 4. It can be seen that the ultimate tensile strength of SiNT is read equal to $13.06 \mathrm{GPa}$ and $4.21 \mathrm{GPa}$, respectively for $(5,5)$ armchair and $(5,0)$ zigzag nanotubes. This shows the substantial impact of the SiNT chirality on the mechanical properties of the structure. Also, another significant point is the discrepancy between mechanical behavior of materials in nanoscale in comparison to the common tensile diagram of structures in macro-scale.

\section{Vibration Analysis of SiNTs}

Vibration analysis and finding natural frequencies of structures are always important and are considered as a significant parameter in designing. Nowadays, due to rapid development of nanotechnology, predicting the intrinsic characteristics of these new nanostructures is attractive to the scientists. In this part three main types of SiNTs free vibration, namely, torsional, longitudinal and transvers vibrational are investigated.

\subsection{Torsional, longitudinal, and transverse vibration}

According to the geometry of a nanotube, torsional vibration analysis is paramount in order to produce nanoprobes and resonators, longitudinal vibration is important due to highly application in piezoelectric and nanocomposites and transverse vibration is one of the most significant parameters in designing and producing. In this section, molecular dynamics simulation is implemented to consider torsional, longitudinal and transverse free vibration behavior of single-walled silicon nanotubes. In these simulations, the Tersoff-Banner potential energy function is used to determine the interatomic forces between the silicon atoms similar to the previous simulation which discussed above. The system is subjected to canonical ensemble at constant temperature $(10 \mathrm{~K})$ which is controlled by Nose-Hoover thermostat. The SWSiNTs are initially relaxed for 50 ps due to minimization of the system's energy in the simulation via the conjugate gradient method to reach the equilibrium state. Thereafter, four layers of atoms are fixed to apply the clamped-free boundary condition, then the proper torsional, elongation and transverse deflections are imposed separately to the free end of SiNT for $3 \mathrm{ps}$, and eventually, the nanotube is released. This allows the
Table 1. Elastic modulus of $(5,5)$ SiNT with length of $5 \mathrm{~nm}$.

\begin{tabular}{cc}
\hline Chirality & $\begin{array}{c}\text { Young's Modulus } \\
(\mathrm{GPa})\end{array}$ \\
\hline$(5,5)$ & 51.08 \\
\hline$(5,0)$ & 46.70 \\
\hline$(6,6)$ & 51.56 \\
\hline$(6,0)$ & 46.81 \\
\hline$(7,7)$ & 51.78 \\
\hline$(7,0)$ & 47.42 \\
\hline$(8,8)$ & 52.26 \\
\hline$(8,0)$ & 47.87 \\
\hline$(9,9)$ & 52.34 \\
\hline$(9,0)$ & 48.11 \\
\hline$(10,10)$ & 52.56 \\
\hline$(10,0)$ & 48.84 \\
\hline
\end{tabular}

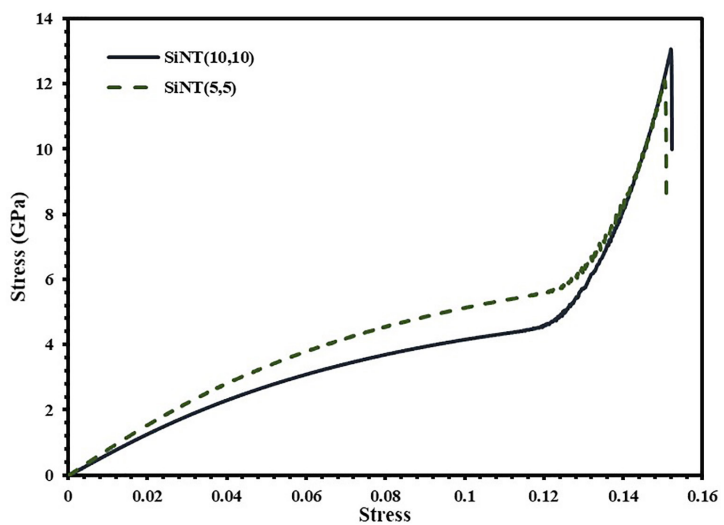

Figure 3. Stress-Strain curve of silicon nanotube armchair $(5,5)$ and $(10,10)$.

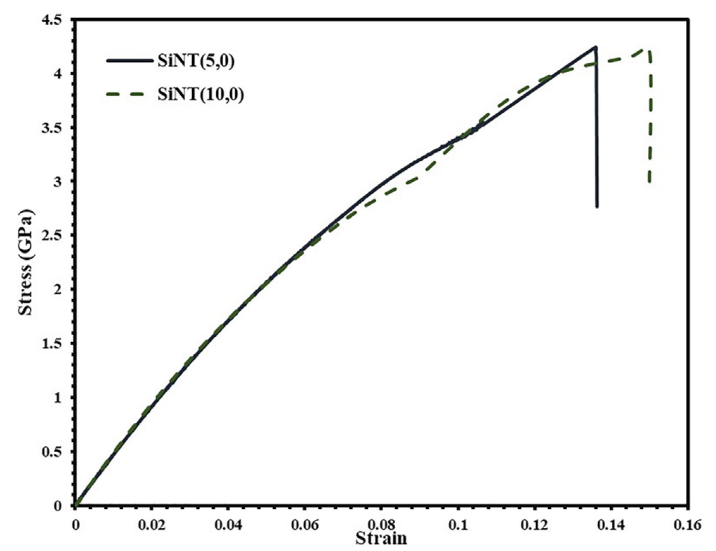

Figure 4. Stress-Strain curve of silicon nanotube zigzag $(5,0)$ and $(10,0)$.

structure to freely vibrate in micro-canonical ensemble. Figures 5, 6, and 7 illustrate a schematic view of the clampedfree SiNTs and the corresponding initial torsional, longitudinal and transverse deformations, respectively. The SiNT natural frequencies of the vibration are found by fitting a sinusoidal curve to the variation of system kinetic energy during the free vibration. The equation below is used to define that trigonometric function. 
(a)
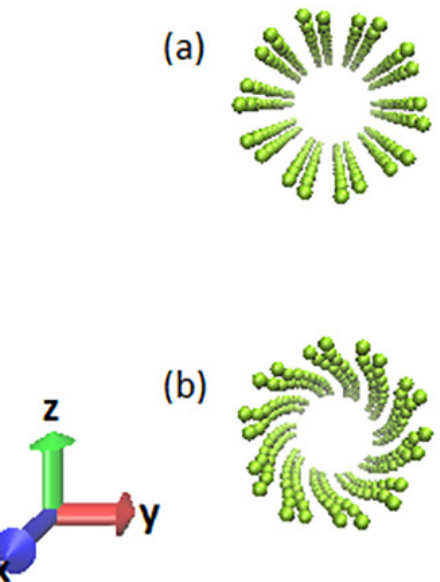

(b)

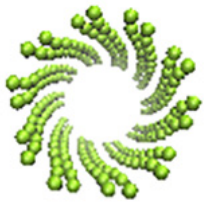

Figure 5. Schematic view of an armchair SWSiNT with C-F boundary conditions (a) before torsional deformation and (b) after initial torsional deformation.

(a)

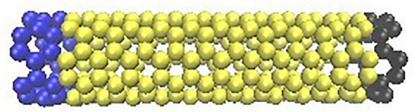

(b)
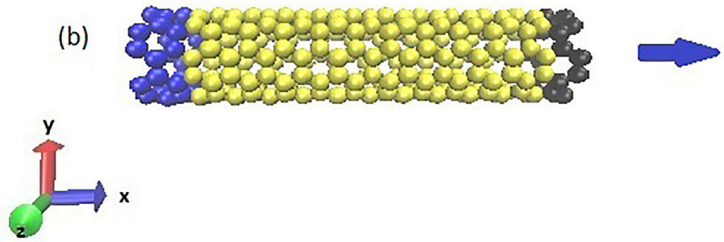

Figure 6. Schematic view of an armchair SWSiNT with C-F boundary conditions (a) relaxed and (b) after initial longitudinal exertion.

(a)
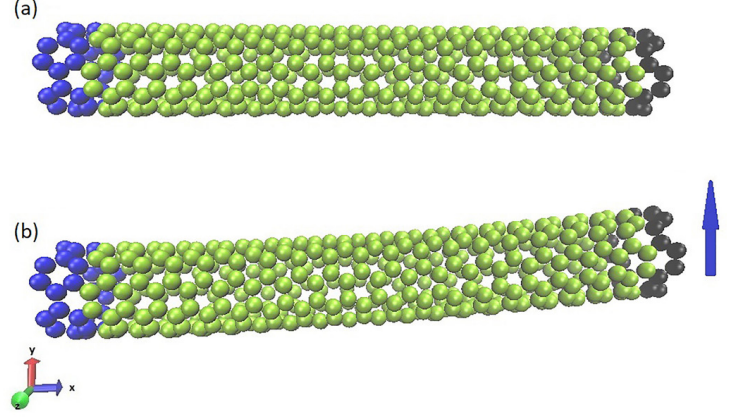

Figure 7. Schematic view of an armchair SWSiNT with C-F boundary conditions (a) minimized energy and (b) after initial transverse deflection.

$$
f(t)=a \sin (\omega t+c)
$$

where $\omega$ denotes the angular natural frequency, $c$ is constant phase of the sine wave and $t$ is the time. Figure 8 demonstrates a representative of curve fitting to the variations of nanotube kinetic energy for a period of 400 ps during free vibration. The SiNTs natural frequencies of the free torsional, longitudinal and transverse vibrations are respectively illustrated for different diameters and chirality in Table 2 .

Since such analysis is carried out for the first time for single-walled silicon nanotubes, to check the validity and accuracy of the current study, the results obtained from molecular dynamics simulation are compared with those obtained from the continuum mechanics solution. To determine the fundamental frequencies in continuum mechanics, the silicon nanotube is assumed as a simple rod with clamped-free boundary conditions which presents orthotropic behavior. Therefore, the first natural frequency of the free torsional vibration can be obtained by using Equation $6^{45}$

$$
f=\frac{1}{4 l} \sqrt{\frac{G}{\rho}}
$$

where $f$ denotes the fundamental frequency, $G$ is the shear modulus, $l$ is the length, and $\rho$ denotes the density. The density is determined using molecular dynamics simulation as follows:

$$
\rho=\frac{M}{V}=\frac{n \times m}{\pi D h l}
$$

where $M$ is the total mass of the SiNT comprises of $n$ atoms with the mass of $m$. Also, $V$ is the volume of the nanotube. Moreover, the shear modulus of the nanotube in the Equation 6 is determined as:

$$
G=\frac{E}{(1+v)}
$$

In above equation, the Young's modulus $(E)$ is calculated by MD for each SWSiNT, and $v$ is the Poisson's ratio determined based on the relative alteration of the nanotube diameter in the transverse direction with respect to the axial axis using Equation 9.

$$
v=-\frac{d_{(\varepsilon)}-d_{0}}{d_{0}}
$$

where $d_{(\varepsilon)}$ denotes the tube diameter at strain $\varepsilon$, and $d_{0}$ is the diameter at the initial state (equilibrium) as shown in Figure 9.

Furthermore, the continuum mechanics solution for the longitudinal and transverse first natural frequency of clamped rods are found using Equations 10 and 1166-47:

$$
f=\frac{1}{4 l} \sqrt{\frac{E}{\rho}}
$$

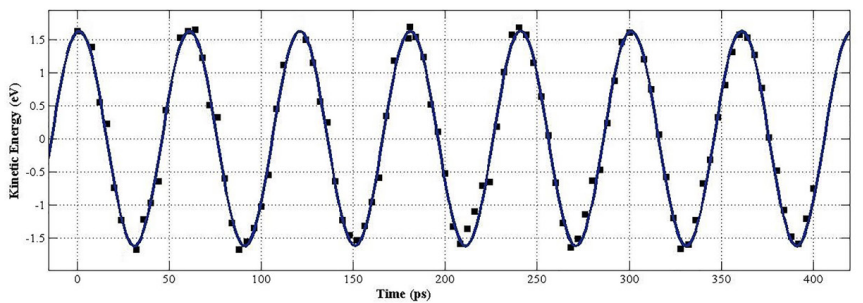

Figure 8. Curve fitting to the profile of system kinetic energy for a period of $400 \mathrm{ps}$ during torsional free vibration. 


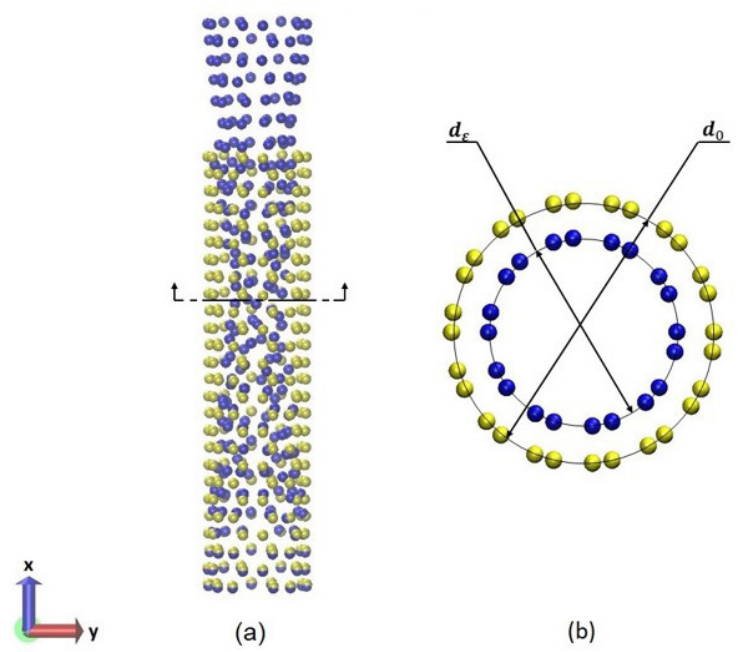

Figure 9. (a) schematic view of a SWSiNT in equilibrium state (Yellow atoms) and Strained state (blue atoms) (b) Middle Cross section view, d_ $((\varepsilon))$ and d_ 0 denote diameter of nanotube in strained state and initial state respectively.

$$
f=\frac{\beta^{2}}{2 \pi l^{2}} \sqrt{\frac{E I}{\rho A}}
$$

where $I$ is the second moment of area, $A$ is the cross-sectional area of nanotube, $\beta$ is approximately equal to $0.5 \pi, l$ is the length and $E$ is the Young's modulus

For comparison study in Table 2, the both of armchair and zigzag nanotubes with length of $5 \mathrm{~nm}$ are modeled. As it can be seen in this table, a good agreement is found between the results calculated from the two different methods. This verifies the accuracy and reliability of the MD model. It is worth noting that the continuum mechanics solution cannot be individually used to predict the frequency of vibration in atomistic level as the Young's modulus is unknown. In the next step, the influence of length on the natural frequency of torsional and transverse vibration of Si nanotubes is studied in Table 3. According to the results, it is observed that chirality and diameter have small effect on the natural frequency of the free vibration, while the length shows a significant impact on this parameter. It is seen that the natural frequency decreases by increasing the length of SiNTs. Also, the zigzag

Table 2. Comparison between torsional, longitudinal and transverse vibration frequencies (THz) of SiNT with $5 \mathrm{~nm}$ length predicted by continuum mechanics and molecular dynamics simulation for different chirality and diameters.

\begin{tabular}{|c|c|c|c|c|c|c|}
\hline \multirow[t]{2}{*}{ Chirality } & \multicolumn{2}{|c|}{$\begin{array}{c}\text { Torsional } \\
\text { Natural Frequencies } \\
(\mathrm{THz})\end{array}$} & \multicolumn{2}{|c|}{$\begin{array}{c}\text { Longitudinal } \\
\text { Natural Frequencies } \\
(\mathrm{THz})\end{array}$} & \multicolumn{2}{|c|}{$\begin{array}{c}\text { Transverse } \\
\text { Natural Frequencies } \\
\text { (THz) }\end{array}$} \\
\hline & MD & $\begin{array}{l}\text { Continuum } \\
\text { Mechanics }\end{array}$ & MD & $\begin{array}{l}\text { Continuum } \\
\text { Mechanics }\end{array}$ & MD & $\begin{array}{l}\text { Continuum } \\
\text { Mechanics }\end{array}$ \\
\hline$(5,5)$ & 0.6381 & 0.62356 & 1.0682 & 0.97375 & 0.1876 & 0.16916 \\
\hline$(5,0)$ & 0.6033 & 0.58714 & 0.9461 & 0.91714 & 0.0995 & 0.09419 \\
\hline$(6,6)$ & 0.6516 & 0.63576 & 1.0763 & 0.99309 & 0.2151 & 0.20562 \\
\hline$(6,0)$ & 0.6246 & 0.60438 & 0.9761 & 0.94408 & 0.1177 & 0.11451 \\
\hline$(7,7)$ & 0.6589 & 0.64255 & 1.0796 & 1.00374 & 0.2424 & 0.24137 \\
\hline$(7,0)$ & 0.6212 & 0.61222 & 0.9951 & 0.95642 & 0.1385 & 0.13408 \\
\hline$(8,8)$ & 0.6637 & 0.64703 & 1.0804 & 1.01072 & 0.2672 & 0.26608 \\
\hline$(8,0)$ & 0.6258 & 0.61637 & 1.0075 & 0.96281 & 0.1591 & 0.15324 \\
\hline$(9,9)$ & 0.6659 & 0.64930 & 1.0828 & 1.01151 & 0.2881 & 0.28773 \\
\hline$(9,0)$ & 0.6286 & 0.62154 & 1.0159 & 0.97087 & 0.1772 & 0.17305 \\
\hline$(10,10)$ & 0.6664 & 0.65272 & 1.0843 & 1.01966 & 0.3052 & 0.30207 \\
\hline$(10,0)$ & 0.6308 & 0.62821 & 1.0211 & 0.98129 & 0.1904 & 0.18844 \\
\hline
\end{tabular}

Table 3. Natural frequencies (THz) of free vibration due to torsional and transverse excitation of armchair $(5,5)$ and zigzag $(5,0)$ SiNT with different lengths calculated by MD simulations.

\begin{tabular}{cccc}
\hline \multirow{2}{*}{$\begin{array}{c}\text { Length } \\
(\mathrm{nm})\end{array}$} & Chirality & $\begin{array}{c}\text { Torsional } \\
\text { Natural Frequencies (THz) }\end{array}$ & $\begin{array}{c}\text { Transverse } \\
\text { Natural Frequencies (THz) }\end{array}$ \\
\hline \multirow{2}{*}{5} & $(5,5)$ & 0.6381 & 0.18766 \\
\cline { 2 - 3 } & $(5,0)$ & 0.6033 & 0.09953 \\
\cline { 2 - 3 } & $(5,5)$ & 0.5302 & 0.12212 \\
\hline \multirow{2}{*}{7} & $(5,0)$ & 0.5078 & 0.07580 \\
\hline \multirow{2}{*}{8} & $(5,5)$ & 0.4277 & 0.09773 \\
\hline \multirow{2}{*}{8} & $(5,0)$ & 0.4218 & 0.05985 \\
\hline \multirow{2}{*}{9} & $(5,5)$ & 0.3896 & 0.08774 \\
\hline \multirow{2}{*}{10} & $(5,0)$ & 0.3797 & 0.03551 \\
\hline & $(5,5)$ & 0.3444 & 0.06636 \\
\hline & $(5,0)$ & 0.3412 & 0.02434 \\
\hline
\end{tabular}


Table 4. Dynamic Young's modulus (GPa) predicted by MD simulations for different lengths of armchair $(5,5)$ and zigzag $(5,0)$ SiNT.

\begin{tabular}{ccc}
\hline Length (nm) & Chirality & $E(\mathrm{GPa})$ \\
\hline \multirow{2}{*}{5} & $(5,5)$ & 50.064 \\
\cline { 2 - 3 } & $(5,0)$ & 46.544 \\
\cline { 2 - 3 } 6 & $(5,5)$ & 50.075 \\
\cline { 2 - 3 } 7 & $(5,0)$ & 46.636 \\
\cline { 2 - 3 } & $(5,5)$ & 50.099 \\
\hline \multirow{2}{*}{8} & $(5,0)$ & 46.663 \\
\hline \multirow{2}{*}{9} & $(5,5)$ & 51.035 \\
\cline { 2 - 3 } & $(5,0)$ & 46.691 \\
\hline \multirow{2}{*}{10} & $(5,5)$ & 51.088 \\
\cline { 2 - 3 } & $(5,0)$ & 46.725 \\
\hline
\end{tabular}

nanotubes possess a lower natural frequency in comparison to armchair ones as an identical length in view of their lower diameter. According to Table 2, it can be deducted that the natural frequencies predicted by the continuum mechanics theory are less than precised values of MD simulations due to neglection of the bond energies, atomic forces and more importantly by asuming a continuum medium. It means that the continuum mechanics theory predicts a lower stiffness for the SiNTs in comparison to the MD simulations.

In addition, based on the MD simulations results of the longitudinal vibrational analysis, the dynamic Young's modulus can be computed. The dynamic Young's modulus parameter is defined as the ratio of stress to strain during the vibrational simulation. It can be calculated by using the natural frequency in either the transverse or longitudinal modes of vibration. Thus, the dynamic Young's modulus equation is found according to Equation 12:

$$
f=\frac{1}{4 l} \sqrt{\frac{E}{\rho}} \rightarrow E=16 f^{2} l^{2} \rho
$$

where $f$ denotes the natural frequency, $E$ is the dynamic Young's modulus, $l$ is the length, and $\rho$ denotes the density.

In this study, the dynamic Young's modulus parameter is obtained by using the MD simulations results for frequency and density and reported in Table 4 for the longitudinal vibration of armchair $(5,5)$ and zigzag $(5,0)$ SiNTs with different lengths for the first time. This practical design parameter can effectively assist engineers to have a more clear insight of structure response for fabricating nano/micro electromechanical devices when the dynamic behavior is important.

\section{Conclusions}

On the basis of molecular dynamics simulations, the torsional, longitudinal, and transverse vibration and mechanical behavior of single-walled silicon nanotubes are investigated. The simulated model contains geometrical parameters such as the length scale, diameter and chirality in order to be able to study influences of aforementioned factors on the free vibration analysis. The Tersoff-Banner potential parameters are used to represent the bonding interatomic forces between the silicon atoms. The fundamental frequencies of free vibration in each case are given relevant to the different length and diameter for both armchair and zigzag SiNTs. It is found that the length size plays a paramount role in the vibration responses of silicon nanotubes and the results clearly illustrate a counter-wise relation between the natural frequency and the length size. However, the diameter is not an effective parameter to alter natural frequencies. Furthermore, a practical design factor named the dynamic Young's modulus is defined and evaluated in this article for the design purpose of the nanoelectremechanical devices such as probes and gigahertz oscillators when the dynamic behavior is the matter of concern. The molecular dynamic results exhibit that this parameter can be efficiently employed to study the free or forced vibrational behavior of nanotubes. The developed results not only clarify the vibrational behavior of SiNTs as an important nano-structured element, but also assist engineers to have better insights in design of micro/ nanoelectromechanical devices.

\section{References}

1. Lieber CM. One-dimensional nanostructures: chemistry, physics \& applications. Solid State Commun. 1998;107(11):607-16.

2. Iijima S. Helical microtubules of graphitic carbon. Nature. 1991;354(6348):56.

3. Peng Q, Liang C, Ji W, De S. Mechanical properties of g-GaN: a first principles study. Appl Phys, A Mater Sci Process. 2013;113(2):483-90.

4. Huang PH, Kuo JK. Thermal stretching of defective nanowires: the coupled effects of vacancy cluster defects, operating temperature, and wire cross-sectional area. Appl Phys, A Mater Sci Process. 2011;103(4):1083-92.

5. Ressel G, Holec D, Fian A, Mendez-Martin F, Leitner H. Atomistic insights into milling mechanisms in an Fe-Y 203 model alloy. Appl Phys, A Mater Sci Process. 2014;115(3):851-8.

6. Al-Tarawneh KM, Al-Aqtash N. Boron-and nitrogen-doped carbon nanotubes with surface defects: an ab initio study. J Comput Theor Nanosci. 2013;10(6):1446-52.

7. Qu LH, Zhang JM, Xu KW, Ji V. Structural and electronic properties of armchair graphene nanoribbons under uniaxial strain. Physica E. 2014;56:55-8.

8. Kuo JK, Huang PH, Wu WT, Lu CM. Mechanical and fracture behaviors of defective silicon nanowires: combined effects of vacancy clusters, temperature, wire size, and shape. Appl Phys, A Mater Sci Process. 2014;114(4):1247-56.

9. Murmu T, McCarthy MA, Adhikari S. In-plane magnetic field affected transverse vibration of embedded single-layer graphene sheets using equivalent nonlocal elasticity approach. Compos Struct. 2013;96:57-63.

10. Arani AG, Rahmani R, Arefmanesh A, Golabi S. Buckling analysis of multi-walled carbon nanotubes under combined loading considering the effect of small length scale. J Mech Sci Technol. 2008;22(3):429-39.

11. Hajian S, Zhang X, Maddipatla D, Narakathu BB, Hanson AJ, Blair RG, et al. Development of a fluorinated graphene-based flexible humidity sensor. In: Proceedings of the 2019 IEEE International Conference on Flexible and Printable Sensors and Systems (FLEPS); 2019 July 7-10; Glasgow. Proceedings. USA: IEEE; 2019. p. 1-3.

12. Ansari R, Shahabodini A, Rouhi H. A thickness-independent nonlocal shell model for describing the stability behavior of carbon nanotubes under compression. Compos Struct. 2013;100:323-31.

13. Natsuki T, Matsuyama N, Shi JX, Ni QQ. Vibration analysis of nanomechanical mass sensor using carbon nanotubes 
under axial tensile loads. Appl Phys, A Mater Sci Process. 2014;116(3):1001-7.

14. Hwu C, Yeh YK. Explicit expressions of mechanical properties for graphene sheets and carbon nanotubes via a molecular-continuum model. Appl Phys, A Mater Sci Process. 2014;116(1):125-40.

15. Ansari R, Sahmani S, Arash B. Nonlocal plate model for free vibrations of single-layered graphene sheets. Phys Lett A. 2010;375(1):53-62.

16. Georgantzinos SK, Anifantis NK. Vibration analysis of multiwalled carbon nanotubes using a spring-mass based finite element model. Comput Mater Sci. 2009;47(1):168-77.

17. Liu B, Huang Y, Jiang H, Qu S, Hwang KC. The atomic-scale finite element method. Comput Methods Appl Mech Eng. 2004;193(17-20):1849-64.

18. Giannopoulos GI, Kontoni DP, Georgantzinos SK. Efficient FEM simulation of static and free vibration behavior of single walled boron nitride nanotubes. Superlattices Microstruct. 2016;96:111-20.

19. Georgantzinos SK, Giannopoulos GI, Anifantis NK. On the coupling of axial and shear deformations of single-walled carbon nanotubes and graphene: a numerical study. Proc Inst Mech Eng, Part N, Nanoeng Nanosyst. 2010;224(4):163-72.

20. Georgantzinos SK, Giannopoulos GI, Anifantis NK. An efficient numerical model for vibration analysis of single-walled carbon nanotubes. Comput Mech. 2009;43(6):731-41.

21. Legoas SB, Coluci VR, Braga SF, Coura PZ, Dantas SO, Galvao DS. Molecular-dynamics simulations of carbon nanotubes as gigahertz oscillators. Phys Rev Lett. 2003;90(5):055504.

22. Setoodeh AR, Badjian H, Jahromi HS. Atomistic study of mono/ multi-atomic vacancy defects on the mechanical characterization of boron-doped graphene sheets. J Mol Model. 2017;23(1):2.

23. Guo W, Guo Y, Gao H, Zheng Q, Zhong W. Energy dissipation in gigahertz oscillators from multiwalled carbon nanotubes. Phys Rev Lett. 2003;91(12):125501.

24. Liu P, Zhang YW, Lu C. Oscillatory behavior of gigahertz oscillators based on multiwalled carbon nanotubes. J Appl Phys. 2005;98(1):014301.

25. Zhang YY, Wang CM, Xiang Y. A molecular dynamics investigation of the torsional responses of defective singlewalled carbon nanotubes. Carbon. 2010;48(14):4100-8.

26. Khademolhosseini F, Phani AS, Nojeh A, Rajapakse N. Nonlocal continuum modeling and molecular dynamics simulation of torsional vibration of carbon nanotubes. IEEE Trans NanoTechnol. 2011;11(1):34-43.

27. Ansari R, Rouhi H, Sahmani S. Calibration of the analytical nonlocal shell model for vibrations of double-walled carbon nanotubes with arbitrary boundary conditions using molecular dynamics. Int J Mech Sci. 2011;53(9):786-92.

28. Setoodeh AR, Badjian H. Mechanical behavior enhancement of defective graphene sheet employing boron nitride coating via atomistic study. Mater Res Express. 2017;4(12):125019.
29. Badjian H, Setoodeh AR. Improved tensile and buckling behavior of defected carbon nanotubes utilizing boron nitride coating-A molecular dynamic study. Physica B. 2017;507:156-63.

30. Bahel A, Ramakrishna MV. Structure of the Si 12 cluster. Phys Rev B. 1995;51(19):13849.

31. Menon M, Richter E. Are quasi-one dimensional structures of Si stable? Phys Rev Lett. 1999;83(4):792.

32. Fagan SB, Mota R, Baierle RJ, Paiva G, Da Silva AJ, Fazzio A. Stability investigation and thermal behavior of a hypothetical silicon nanotube. J Mol Struct THEOCHEM. 2001;539(13):101-6.

33. Kang JW, Hwang HJ. Mechanical deformation study of copper nanowire using atomistic simulation. Nanotechnology. 2001;12(3):295.

34. Lourie O, Cox DM, Wagner HD. Buckling and collapse of embedded carbon nanotubes. Phys Rev Lett. 1998;81(8):1638.

35. Jeng YR, Tsai PC, Fang TH. Effects of temperature, strain rate, and vacancies on tensile and fatigue behaviors of silicon-based nanotubes. Phys Rev B. 2005;71(8):085411.

36. Vellayappan MV, Balaji A, Subramanian AP, John AA, Jaganathan SK, Murugesan S, et al. Tangible nanocomposites with diverse properties for heart valve application. Sci Technol Adv Mater. 2015;16(3):033504.

37. Tersoff J. New empirical model for the structural properties of silicon. Phys Rev Lett. 1986;56(6):632.

38. Tersoff J. New empirical approach for the structure and energy of covalent systems. Phys Rev B. 1988;37(12):6991.

39. Dodson BW. Development of a many-body Tersoff-type potential for silicon. Phys Rev B. 1987;35(6):2795.

40. Tersoff JJ. Modeling solid-state chemistry: interatomic potentials for multicomponent systems. Phys Rev B. 1989;39(8):5566.

41. Tersoff J. Erratum: Modeling solid-state chemistry: Interatomic potentials for multicomponent systems. Phys Rev B. 1990;41(5):3248.

42. Plimpton S. Fast parallel algorithms for short-range molecular dynamics. J Comput Phys. 1995;117(1):1-9.

43. Setoodeh A, Attariani H, Jahanshahi M. Mechanical properties of silicon-germanium nanotubes under tensile and compressive loadings. Journal of Nano Research. 2011;15:105-14.

44. Swope WC, Andersen HC, Berens PH, Wilson KR. A computer simulation method for the calculation of equilibrium constants for the formation of physical clusters of molecules: application to small water clusters. J Chem Phys. 1982;76(1):637-49.

45. Weaver W Jr, Timoshenko SP, Young DH. Vibration problems in engineering. Hoboken: John Wiley \& Sons; 1990.

46. Inman DJ, Singh RC. Engineering vibration. Englewood Cliffs, NJ: Prentice Hall; 1994.

47. Morse PM, Acoustical Society of America, American Institute of Physics. Vibration and sound. New York: McGraw-Hill; 1948. 